\title{
Variación de las propiedades termofísicas de trucha arco iris (Oncorhynchus mykiss) durante el rigor mortis
}

\author{
Changes of thermophysical properties of rainbow trout \\ (Oncorhynchus mykiss) during rigor mortis
}

Fabiola Olivares $^{1}$; Domingo Sánchez ${ }^{2}$

\begin{abstract}
Resumen
Después de la captura y muerte del pez, este experimenta una serie de cambios bioquímicos que afectan su composición y dan lugar al proceso de deterioro influyendo en la caracterización de las propiedades termofísicas del músculo. La velocidad de deterioro varía según las especies, dependiendo de diversos factores tales como el tamaño, estado fisiológico, alimentación, método de captura, temperatura de almacenamiento, entre otros. Con la finalidad de determinar el comportamiento de las principales propiedades termofísicas durante el desarrollo del rigor mortis de trucha arco iris (Oncorhynchus mykiss), se determinó experimentalmente la variación del calor específico (Cp), difusión térmica (a), densidad $(\mathrm{r})$ y conductividad térmica $(\mathrm{k})$ en diferentes intervalos de tiempo. Cuando se evaluaron las fases del rigor mortis, el Índice de Rigor (IR) alcanzó el $86 \%$ a las 2 horas de almacenamiento $\left(14 \pm 1^{\circ} \mathrm{C}\right.$ ) y el $100 \%$ a las 4 horas, luego de las cuales decreció abruptamente. El calor específico $(\mathrm{Cp})$ decreció a medida que transcurrió el tiempo de almacenamiento, reportando valores que fluctúan entre 7,89 y $0,74 \mathrm{cal} / \mathrm{g}{ }^{\circ} \mathbf{C}$. Durante las 2 primeras horas de rigor mortis (pre rigor), los valores de la difusión térmica (a) y la densidad (r) presentaron una caída brusca, para posteriormente evidenciar un comportamiento ascendente logrando alcanzar durante el pleno rigor valores de 3,32 x $10^{-7} \mathrm{~m}^{2} / \mathrm{s}$ y $1320 \mathrm{~kg} / \mathrm{m}^{3}$ respectivamente. La conductividad térmica (k) presentó una tendencia decreciente, pero a partir de las 2 horas de almacenamiento no se observaron mayores variaciones $\left(3,46-3,64 \mathrm{~W} / \mathrm{m}^{\circ} \mathbf{C}\right)$. Por lo expuesto observamos que los cambios post mortem influyen en la variación de las propiedades termofísicas del músculo de trucha arco iris durante el almacenamiento, bajo las condiciones estudiadas $\left(14 \pm 1^{\circ} \mathrm{C}, 6\right.$ horas $)$.
\end{abstract}

Palabras clave: propiedades termofísicas; rigor mortis; trucha arco iris.

\begin{abstract}
After the capture and death of the fish; it undergoes a series of biochemical changes which affect its composition leading to the deterioration process influencing in the characterization of the thermophysical properties of the muscle. The rate of deterioration varies among species, depending on various factors such as size, physiological condition, nutrition, catching method, storage temperature, among others. In order to determine the behavior of the main thermophysical properties during the development of rigor mortis of rainbow trout (Oncorhynchus mykiss), the variation of specific heat $(\mathrm{Cp})$, thermal diffusivity (a), density ( $\mathrm{r}$ ) and thermal conductivity (k) were determined experimentally at different intervals of time. When rigor mortis phases were evaluated, Rigor Index (IR) reached $86 \%$ after 2 hours of storage $\left(14 \pm 1{ }^{\circ} \mathrm{C}\right)$ and $100 \%$ at 4 hours, after which decreased abruptly. The specific heat $(\mathrm{Cp})$ decreased as storage time passed, reporting values between 7,89 and $0,74 \mathrm{cal} / \mathrm{g}{ }^{\circ} \mathrm{C}$. During the first 2 hours of rigor mortis (pre rigor), the values of thermal diffusivity (a) and density (r) presented a sudden fall and later showed an ascending behavior reaching during full rigor values of $3,32 \times 10^{-7} \mathrm{~m}^{2} / \mathrm{s}$ and $1320 \mathrm{~kg} / \mathrm{m}^{3}$ respectively. The thermal conductivity (k) showed a decreasing tendency and after 2 hours of storage no major variations were observed $\left(3,46-3,64 \mathrm{~W} / \mathrm{m}{ }^{\circ} \mathrm{C}\right)$. Post mortem changes affect the behavior of the thermophysical properties of rainbow trout muscle during storage under the studied conditions $\left(14 \pm 1^{\circ} \mathrm{C}, 6\right.$ hours).
\end{abstract}

Keywords: thermophysical properties; rigor mortis; rainbow trout.

1. Facultad de Pesquería, Universidad Nacional Agraria La Molina, Lima (Perú). Email: folivares@lamolina.edu.pe

2. Facultad de Pesquería, Universidad Nacional Agraria La Molina, Lima (Perú). Email: dsa@lamolina.edu.pe 


\section{Introducción}

La calidad de la carne de pescado, reflejada principalmente en la firmeza del músculo, es uno de los factores más importantes al momento de aplicar un proceso tecnológico. Algunos estudios demuestran que, con cierta frecuencia, la carne de pescado se ablanda después de 24 horas de almacenamiento en frío y que dicho comportamiento podría estar relacionado con las resolución del rigor mortis (Toyohara y Shimizu, 1988; Oka, Ohno y Ninomuya, 1990; Ando, Nishiyabu, Tsukamasa y Makinodan, 1991; Mochizuki y Sato, 1996). La mayor parte de estos trabajos han sido realizados principalmente en peces marinos y son pocos los relacionados con especies de agua dulce (Ma y Yamanaka, 1991; Ando et al., 1999). Sin embargo, para explicar el fenómeno del ablandamiento post mortem encontrado en especies sometidas a cultivo, se han planteado diversas teorías. Hatae, Tamari, Miyanaga y Matsumoto (1985); Tachibana, Misima y Tsuchimoto (1993) proponen, simplemente, que el ablandamiento sucede más temprano en especies de cultivo que en individuos de la misma especie provenientes de ambiente natural.

Se ha considerado que los cambios ocurridos con relación a las propiedades físicas de la carne de pescado, son causados más por cambios en las estructuras del tejido muscular que por cambios en los componentes de las proteínas (Hatae et al., 1985). Ando et al. (1999) consideran que los peces de cultivo, al estar bien alimentados, poseen una tasa de rotación de proteína o turnover mucho más elevada que peces de ambiente natural, lo que podría originar una actividad de proteólisis elevada en el músculo de los peces de cultivo.

Dado que muchas etapas en el procesamiento y conservación de pescado y productos de la pesca implican la transferencia de calor, es importante comprender las propiedades termofísicas de los mismos. El calor específico (Cp) es el que determina la cantidad de calor requerido para aumentar la unidad de temperatura por unidad de masa de la sustancia, mientras que la conductividad térmica (k) se define como la capacidad de un material para conducir el calor. Ambas propiedades dependen en gran medida de la temperatura y la composición química (Wallapapan, Sweat, Diehl y Engler, 1986; Choi y Okos, 1986). La difusión térmica (a) mide la capacidad de un material para conducir energía térmica y puede calcularse indirectamente a partir de la conductividad térmica (k), la densidad (r) y el calor específico (Cp) (Meffert, 1984; Kreith y Bohn, 2001).

$\mathrm{Si}$ bien las propiedades termofísicas y los modelos matemáticos aplicados para su cálculo han sido estudiados por diferentes investigadores (Kumbhar, Agarwal y Das, K., 1981; Lind, 1991; Radhakrishnan, 1997; Banga, Alonso, Gallardo y Pérez-Martin, 1993; Ochoa, Amézquita y Chejne, 2006), la información disponible es dispersa, fraccionada y tiene un alto grado de variación, lo que dificulta el uso de los valores publicados y de los modelos propuestos. Por otro lado, son casi inexistentes los estudios que relacionan el comportamiento de las propiedades termofísicas del músculo durante el almacenamiento y desarrollo del rigor mortis, sin embargo los datos de dichas propiedades son necesarios para los cálculos de ingeniería y diseño de procesos de enfriamiento y calentamiento principalmente.

El presente estudio tiene por objetivo determinar y evaluar la variación de las principales propiedades termofísicas: calor específico (Cp), difusión térmica (a), densidad (r) y conductividad térmica $(\mathrm{k})$ de trucha arco iris (Oncorhynchus mykiss) durante el desarrollo del rigor mortis.

\section{Materiales y métodos}

\section{Lugar de ejecución}

El presente trabajo de investigación se desarrolló en el Laboratorio de Piscifactoría y en las instalaciones de la empresa Los Andes S.A., ubicada en la provincia de Canta, Lima (Perú).

Materia prima.

Se utilizó truchas arco iris (Oncorhynchus mykiss) capturadas directamente de las pozas del centro de producción de piscifactoría de Los Andes. La longitud y peso de los individuos fue de $25,15 \pm 1,32 \mathrm{~cm}$ y $0,20 \pm 0,02$ $\mathrm{kg}$, respectivamente. Una vez producida la muerte por asfixia, las 27 truchas fueron colocadas en una caja térmica (cooler) y mantenidas a $14 \pm 1{ }^{\circ} \mathrm{C}$ hasta la determinación del índice de rigor (IR). Para evaluar la variación de las propiedades termofísicas a esa temperatura $\left(14 \pm 1{ }^{\circ} \mathrm{C}\right)$, los individuos utilizados para el IR fueron eviscerados, descabezados y fileteados. Todas las determinaciones se realizaron por triplicado.

\section{Preparación de la muestra}

Los filetes fueron troceados y picados con cuchillo hasta su completa homogeneización. Para cada evaluación a diferentes intervalos de tiempo se utilizó $240 \pm 2$ g de muestra homogeneizada.

\section{Métodos}

\section{Determinación del índice de rigor (IR)}

El método utilizado fue el descrito por Bito, citado por Iwamoto, Yamanaka, Watabe y Hashimoto (1987). El pescado después de muerto fue colocado horizontalmente sobre una tabla, sobresaliendo libremente la mitad de la longitud total del cuerpo $\left(\mathrm{L}_{0}\right)$. En diferentes intervalos de tiempo (horas), se midió la distancia desde la proyección de la línea horizontal de la tabla hasta la base de la aleta caudal del pescado (L). La temperatura durante la evaluación de las muestras fue de $14 \pm 1^{\circ} \mathrm{C}$. Los resultados se expresan en porcentaje (\%) y se realizaron al menos tres repeticiones. Para el cálculo del IR se utilizó la siguiente expresión:

Donde:

$$
I R=\left[\frac{\left(L_{0}-L\right)}{L_{0}}\right] \times 100(\%)
$$

$\mathrm{L}_{0}=$ Valor inicial inmediatamente después de muerto $(\mathrm{cm})$ $\mathrm{L}=$ Distancia desde la proyección de la línea horizontal hasta la base de la aleta caudal $(\mathrm{cm})$ 


\section{Determinación del calor específico (Cp)}

Se utilizó el método calorimétrico y el protocolo es descrito por Sánchez y Olivares (2007). Se determinó el $\mathrm{Cp}$ a diferentes intervalos de tiempo (horas) y los resultados se expresaron en $\mathrm{cal} / \mathrm{g}{ }^{\circ} \mathrm{C}$. Se realizaron tres repeticiones y para el cálculo se utilizó la siguiente expresión:

$$
C p=\frac{C m}{\text { Masa de la muestra }}\left(\frac{\mathrm{cal}}{\mathrm{g}^{\circ} \mathrm{C}}\right)
$$

Donde:

$\mathrm{Cm}=$ Capacidad calorífica de la muestra $\left(\mathrm{cal} /{ }^{\circ} \mathrm{C}\right)$

\section{Determinación de la difusión térmica (a)}

El método utilizado fue el sugerido por Ibarz, Barbosa, Garza y Gimeno (2000) y descrito por Sánchez y Olivares (2007). La difusión térmica se determinó gráficamente a diferentes intervalos de tiempo (horas) durante el almacenamiento, utilizando la ecuación (4), en donde el valor de a se igualó al término equivalente a la pendiente de la recta obtenida. Se realizaron tres repeticiones y los resultados se expresan en $\mathrm{m}^{2} / \mathrm{s}$.

$$
Y_{c f}=\frac{T-T_{\infty}}{T_{o}-T_{\infty}}
$$

$\log _{c f}=\log 2,040-\left[\left(\frac{1,017}{a^{2}}+\frac{2,512}{r_{c}^{2}}\right)\right] t$

$\mathrm{Y}_{\mathrm{cf}} \quad=$ Relación adimensional de temperatura

$\mathrm{T}=$ Temperatura variable a los tiempos respectivos $\left({ }^{\circ} \mathrm{C}\right)$

$\mathrm{T}_{\mathrm{o}} \quad=$ Temperatura inicial $\left({ }^{\circ} \mathrm{C}\right)$

$\mathrm{T}_{¥} \quad=$ Temperatura del baño maría $\left({ }^{\circ} \mathrm{C}\right)$

$\mathrm{r}_{\mathrm{c}} \quad=$ Radio del cilindro $(\mathrm{m})$

a = Mitad del espesor de la lámina infinita (m)

$\alpha \quad=$ Difusión $\left(\mathrm{m}^{2} / \mathrm{s}\right)$

$\mathrm{t} \quad=$ Tiempo $(\mathrm{h})$

\section{Determinación de la densidad (r)}

La densidad de las muestras fue obtenida de manera indirecta por cálculo, a partir de la determinación de la gravedad específica (Gs). Para el cálculo de Gs se aplicó el método sugerido por Casimir en 1967 y citado por Cárdenas, Córdoba, Cohaila y Gonzales (1979), adaptado a las condiciones del laboratorio. Se realizaron tres repeticiones y se utilizó la siguiente expresión:

$$
G s=\frac{(Z-X)}{[(Y-X)-(W-Z)]}
$$

Donde:

$$
\begin{array}{ll}
\mathrm{X} & =\text { Peso de la botella vacía y seca }(\mathrm{g}) \\
\mathrm{Y} & =\text { Peso de la botella llena de agua }(\mathrm{g}) \\
\mathrm{W} & \text { = Peso de la botella con agua y muestra }(\mathrm{g}) \\
\mathrm{Z} & \text { = Peso de la botella y muestra cuya gravedad va }
\end{array}
$$

a determinarse $(\mathrm{g})$

Para determinar la densidad se utilizó la expresión sugerida por Sigh y Heldman (1998) en la que se relaciona el peso del alimento con el peso del agua, considerando volúmenes iguales de alimento y agua. Los resultados de densidad se expresan en $\mathrm{kg} / \mathrm{m}^{3}$. Para los cálculos se aplicó la siguiente expresión:

$$
G s=\frac{\rho_{s}}{\rho_{w}}=\frac{P_{s}}{P_{w}}
$$

Donde:

$$
\begin{array}{ll}
\rho_{\mathrm{s}} & =\text { Densidad del alimento } \\
\rho_{\mathrm{w}} & =\text { Densidad del agua } \\
\mathrm{P}_{\mathrm{s}} & =\text { Peso específico del alimento } \\
\mathrm{P}_{\mathrm{w}} & =\text { Peso específico del agua }
\end{array}
$$

\section{Determinación de la conductividad térmica $(k)$}

La conductividad térmica de las muestras fue obtenida de manera indirecta por cálculo, conocidos los valores de las demás variables. Se utilizó la expresión sugerida por Carslaw y Jaeger (1959) y citada por Ibarz et al. (2000). Se realizaron tres repeticiones. Los resultados se expresan en $\mathrm{kJ} / \mathrm{s} \mathrm{m}^{\circ} \mathrm{C}$.

$$
\alpha=\frac{K}{\rho C p}
$$

Donde:

$$
\begin{array}{ll}
\mathrm{K} & =\text { Conductividad térmica de la muestra }\left(\mathrm{W} / \mathrm{m}^{\circ} \mathrm{C}\right) \\
\mathrm{a} & =\text { Difusión térmica de la muestra }\left(\mathrm{m}^{2} / \mathrm{s}\right) \\
\mathrm{r} & =\text { Densidad de la muestra }\left(\mathrm{kg} / \mathrm{m}^{3}\right) \\
\mathrm{Cp} & =\text { Calor específico de la muestra }\left(\mathrm{kJ} / \mathrm{kg}{ }^{\circ} \mathrm{C}\right)
\end{array}
$$

\section{Resultados y discusión \\ Índice de rigor (IR) de la materia prima}

En la Fig. 1 se presentan las fases del rigor mortis y la variación del IR de trucha arco iris durante almacenamiento $\left(14 \pm 1 \quad{ }^{\circ} \mathrm{C}\right)$. El rigor mortis se desarrolló inmediatamente después de la muerte del pez, presentando un comportamiento creciente durante las primeras horas de estudio (pre rigor, 0-2 horas), alcanzando la máxima tensión de rigor $(\mathrm{IR}=100 \%)$ a las 4 horas de almacenamiento y que corresponde al desarrollo del pleno rigor. Pasado ese tiempo el IR comenzó a decaer gradualmente (post rigor a partir de las 5 horas); comportamiento previamente reportado por Duran, Erdemli, Karakaya y Yilmaz (2008) en especies de agua dulce almacenadas en condiciones similares de temperatura y por Iwamoto et al. (1987) en especies marinas.

Llerena y Nué (2002) demostraron que la máxima tensión de rigor está relacionada inversamente con la temperatura y tiempo de almacenamiento. Para trucha arco iris almacenada a $5{ }^{\circ} \mathrm{C}$, el pre rigor puede iniciarse hasta 12 horas después de la muerte del animal, mientras que el post rigor entre 48 y 60 horas (Duran et al., 2008). Bertullo (1975) y Suárez, De Francisco, Beirão, 


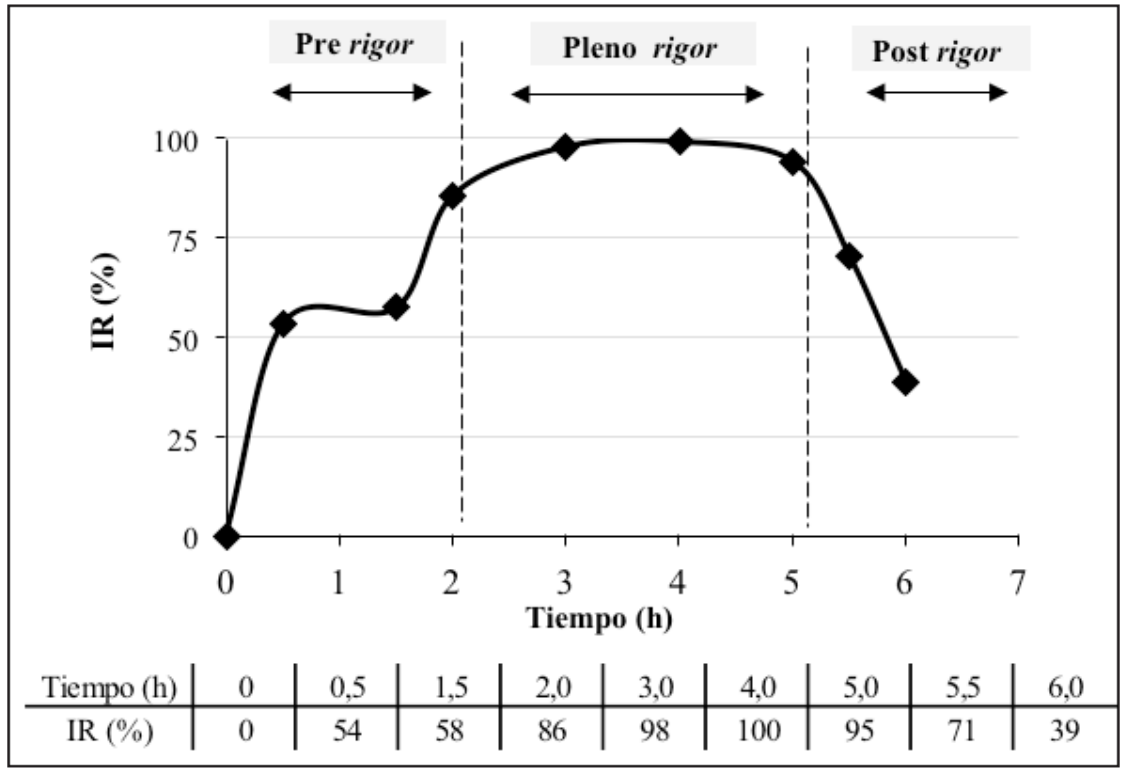

Figura 1. Fases del rigor mortis y variación del índice de rigor (IR) (\%) de trucha arco iris (Oncorhynchus mykiss) durante almacenamiento a $14 \pm 1{ }^{\circ} \mathrm{C}$.

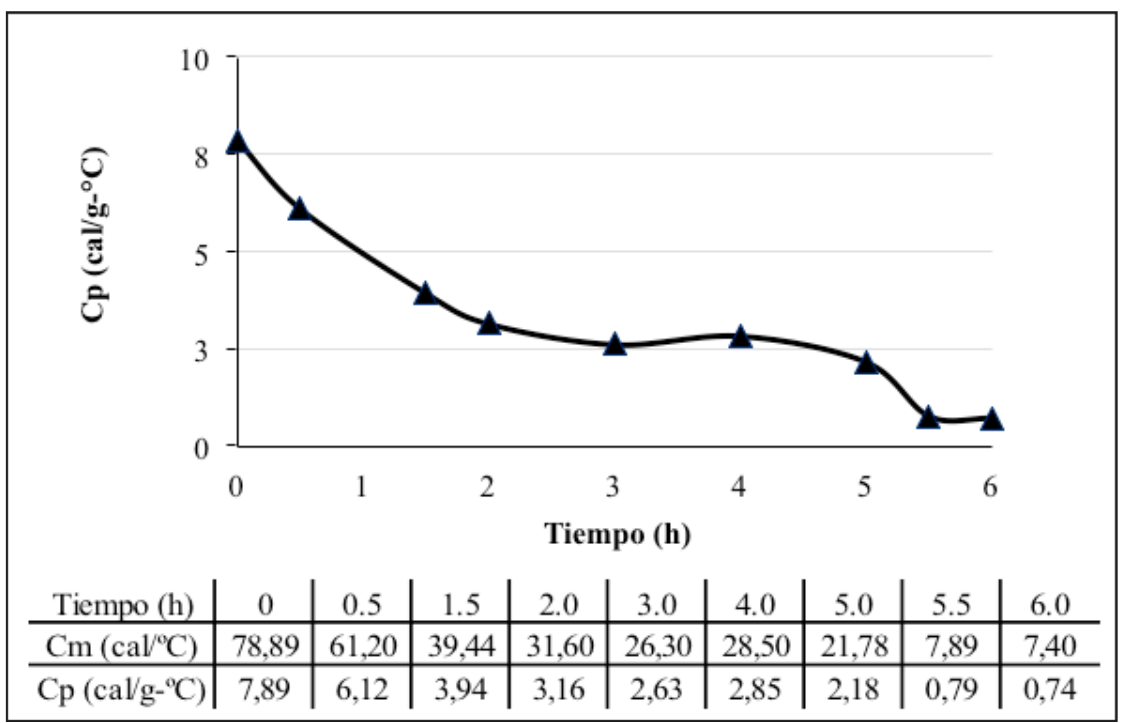

Figura 2. Variación del calor específico $(\mathrm{Cp})\left(\mathrm{cal} / \mathrm{g}{ }^{\circ} \mathrm{C}\right)$ de trucha $\operatorname{arco}$ iris (Oncorhynchus mykiss) durante almacenamiento a $14 \pm 1{ }^{\circ} \mathrm{C}$.

Pardo y Cortés (2007) indican que el desarrollo del rigor mortis no es igual para todas las especies, sugiriendo que dependerá del hábitat del animal (ambiente marino, agua dulce, acuicultura), condiciones de captura y sacrificio (asfixia, aplastamiento) y condiciones de manipulación y almacenamiento (temperatura). En nuestro caso, al realizar el estudio a temperaturas mayores a $10^{\circ} \mathrm{C}$, el rigor mortis se desarrolló más rápidamente que a temperaturas inferiores $\left(\geq 5^{\circ} \mathrm{C}\right)$.

Aunque no se pudo evaluar la textura, se observó ablandamiento de la carne pasadas las cuatro horas de estudio. Al respecto, Ando et al. (1991) al realizar estudios en especies de agua dulce (Cyprinus carpio, Oncorhynchus mykiss), demostraron que el ablandamiento del músculo de los peces es diferente en cada especie y que es un proceso independiente del rigor mortis.

\section{Determinación del calor específico $(C p)$}

En la Fig. 2, se presenta la variación del $\mathrm{Cp}\left(\mathrm{cal} / \mathrm{g}{ }^{\circ} \mathrm{C}\right)$ durante el almacenamiento $\left(14 \pm 1{ }^{\circ} \mathrm{C}\right)$ de trucha arco iris. Se observa una tendencia decreciente del $\mathrm{Cp}$ a medida que avanza el rigor mortis, obteniéndose valores entre 7,89 y $0,74 \mathrm{cal} / \mathrm{g}{ }^{\circ} \mathrm{C}$. Esta disminución se debe principalmente a la caída brusca de la capacidad calórica de la muestra $(\mathrm{Cm})$, tal como se observa en los valores reportados en la Fig. 2.

Radhakrishnan (1997) reporta valores de calor específico para trucha arco iris que oscilan entre 3,1 y $3,8 \mathrm{~kJ} /$ $\mathrm{kg}{ }^{\circ} \mathrm{C}$. Asimismo, establece una relación inversa con la temperatura, asociándola con el contenido de agua 
de la especie. Aunque en el presente estudio no se ha demostrado, es posible que el calor específico varíe al disminuir la capacidad de retención de agua del músculo por efecto de los fenómenos bioquímicos desarrollados en el rigor mortis. Actualmente, se vienen realizando diversos estudios para verificar dicho comportamiento.

\section{Determinación de la difusión térmica $(\alpha)$}

La difusión térmica fue calculada a $70 \pm 2,5^{\circ} \mathrm{C}$ y estuvo en el orden de $10^{-7} \mathrm{~m}^{2} / \mathrm{s}$, tal como se presenta en la Fig. 3 .

Los valores de difusión térmica son raramente encontrados en la literatura, lo que dificulta una comparación directa. Sin embargo, Sánchez y Olivares (2007) reportan para trucha arco iris un valor promedio de $5,82 \times 10^{-8} \mathrm{~m}^{2} / \mathrm{s}$. Durante la evolución del rigor mortis, con excepción de las dos primeras horas en las que se observó una caída brusca $\left(1,12 \times 10^{-7} \mathrm{~m}^{2} / \mathrm{s}\right)$, la difusión térmica tuvo un comportamiento ascendente al iniciar la fase de pleno rigor. Esta disminución inicial coincidió con la fase de pre rigor, en la cual se produce una rigidez en el músculo generando una mayor resistencia al paso del calor. Es así que materiales de a pequeño responden más lentamente a los cambios en su medio y tardan más en alcanzar una nueva condición de equilibrio (Incropera y DeWitt, 2002). El máximo valor de a fue $3,3 \times 10^{-7} \mathrm{~m}^{2} / \mathrm{s}$ y se obtuvo a las 6 horas de almacenamiento (post rigor).

\section{Determinación de la densidad ( $\rho)$}

El comportamiento de la densidad de trucha arco iris durante el almacenamiento $\left(14 \pm 1{ }^{\circ} \mathrm{C}\right)$ se presenta en la Fig. 4.

Sing y Heldman (1998) definen la densidad de un alimento como un indicador que muestra la manera en que la materia está organizada en un cuerpo; por tanto, si la estructura es más compacta, tiene mayor densidad. En nuestro caso, antes de las 2 primeras horas de estudio, se observó un ligero descenso de la densidad, comportamiento que podría estar relacionado con la primera fase del rigor

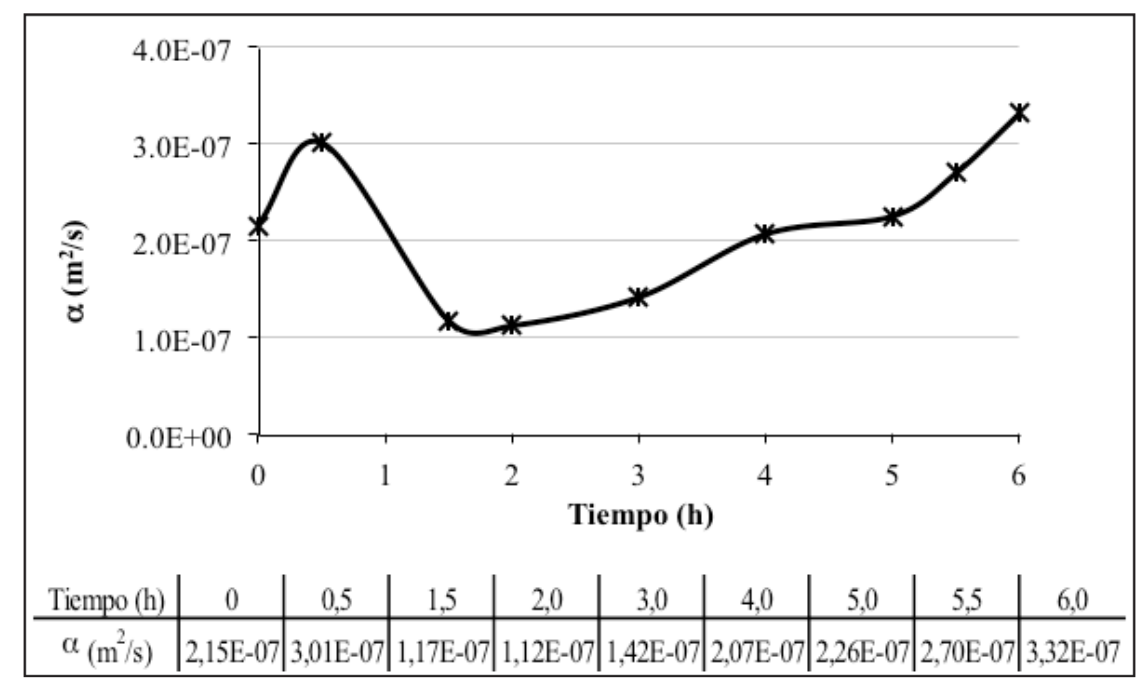

Figura 3. Variación de la difusión térmica (a) $\left(\mathrm{m}^{2} / \mathrm{s}\right)$ de trucha aro iris (Oncorhynchus mykiss) durante almacenamiento a $14 \pm 1^{\circ} \mathrm{C}$.

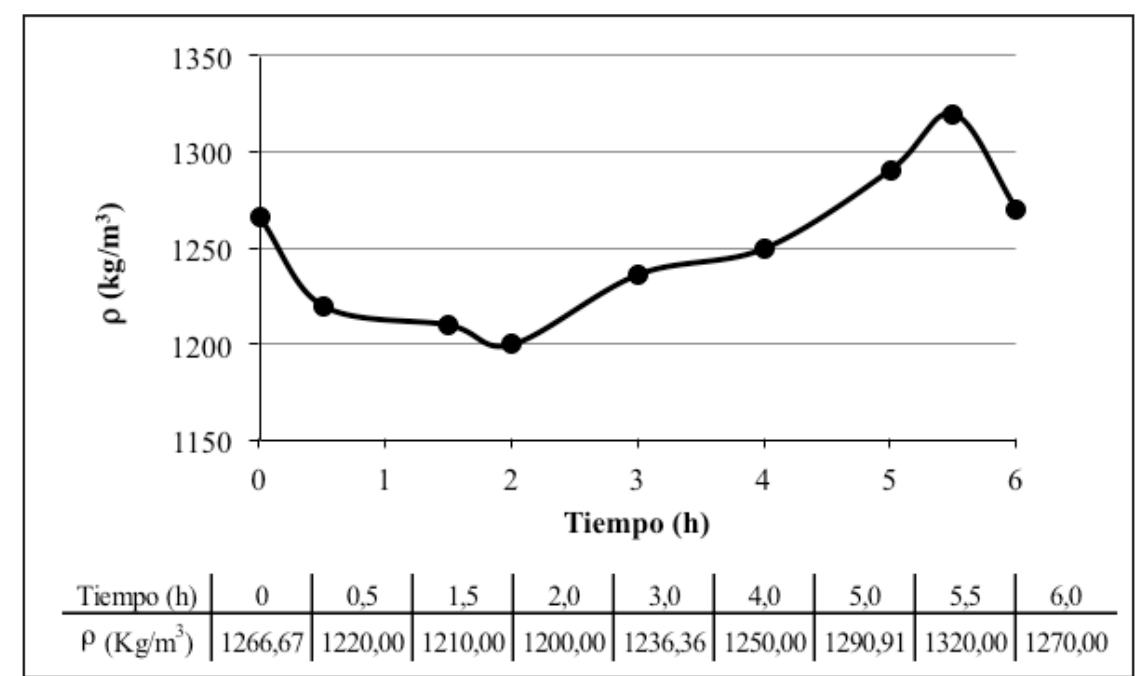

Figura 4. Variación de la densidad $(\mathrm{r})\left(\mathrm{kg} / \mathrm{m}^{3}\right)$ de trucha arco iris (Oncorhynchus mykiss) durante almacenamiento a $14 \pm 1{ }^{\circ} \mathrm{C}$. 


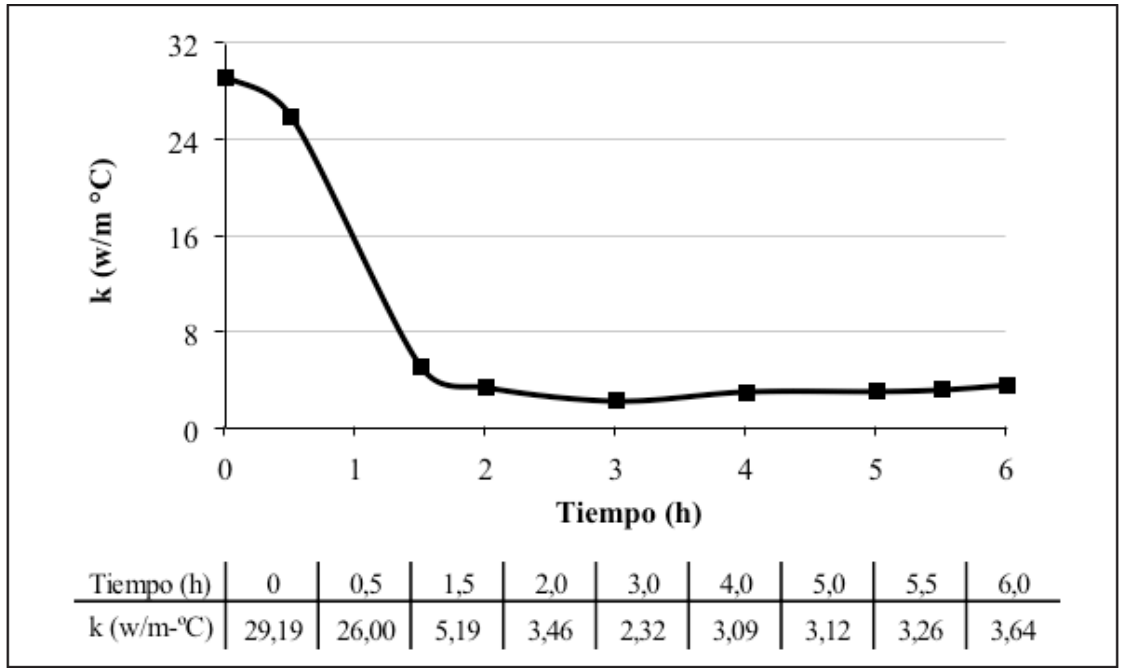

Figura 5. Variación de la conductividad térmica $(\mathrm{k})\left(\mathrm{w} / \mathrm{m}{ }^{\circ} \mathrm{C}\right)$ de trucha arco iris (Oncorhynchus mykiss) durante almacenamiento a $14 \pm 1{ }^{\circ} \mathrm{C}$.

mortis. La pérdida de agua del músculo (“drip”), a partir de las dos horas de almacenamiento, coincidió con el completo desarrollo del rigor mortis y se reflejó en un aumento progresivo de la densidad, propiedad que depende de la relación del peso y volumen. El máximo valor de densidad $\left(1320 \mathrm{~kg} / \mathrm{m}^{3}\right)$ se obtuvo a las 5,5 horas de almacenamiento.

\section{Determinación de la conductividad térmica ( $k$.}

Los valores de conductividad térmica se presentan en la Fig. 5 y oscilan entre 29,19 y $3,64 \mathrm{~W} / \mathrm{m}{ }^{\circ} \mathrm{C}$ dependiendo del tiempo transcurrido desde la muerte del animal. El máximo valor de $\mathrm{k}$ se observó al inicio del experimento $\left(29,19 \mathrm{~W} / \mathrm{m}^{\circ} \mathrm{C}\right)$ y durante la fase de pre rigor y fue a partir de las dos horas de almacenamiento $\left(14 \pm 1^{\circ} \mathrm{C}\right)$ que la conductividad térmica se mantuvo sin mayores variaciones, obteniéndose valores entre 2,32 y $3,64 \mathrm{~W} / \mathrm{m}$ ${ }^{\circ} \mathrm{C}$.

Diversos autores han demostrado que a medida que transcurre el rigor mortis, el músculo de pescado va perdiendo humedad y su capacidad de retención de agua (Suzuki, 1987; Huss, 1988; Tejada, Huidobro y Fouad Mohamed, 2003; Huidobro y Tejada, 1993). Por su parte, Lind (1991) indica que la variación de la conductividad térmica está relacionada con el contenido de agua del alimento mas no con la temperatura de almacenamiento. En nuestro caso, observamos que a medida que transcurre el tiempo de almacenamiento el músculo de la trucha va perdiendo humedad y la conductividad disminuye haciéndose constante a partir de las dos horas de estudio (Fig. 5), comportamiento que coincide con el inicio del pleno rigor.

\section{Conclusiones}

Los cambios que experimenta el pescado durante el desarrollo del rigor mortis, influyen en la variación de las propiedades termofísicas durante el almacenamiento bajo las condiciones estudiadas. Mientras que el calor específico $(\mathrm{Cp})$ y la conductividad térmica $(\mathrm{k})$ presentan un comportamiento decreciente, la densidad (r) y la difusión térmica (a) aumentan a medida que avanza el rigor mortis. En todos los casos, con excepción de la conductividad térmica $(\mathrm{k})$, fue a partir de las 2 horas de almacenamiento que se observó la tendencia final de las propiedades y coincidió con el completo desarrollo del rigor mortis (pleno rigor). En la industria, el cálculo de las diferentes propiedades termofísicas es considerado como una herramienta indispensable para el diseño de los procesos que implican calentamiento o enfriamiento.

\section{Literatura citada}

Ando, M.; Nishiyabu, A.; Tsukamasa, Y. y Makinodan, Y. 1999. Post mortem softening of fish muscle during chilled storage as affected by bleeding. J Food Sci, 64(3): 423-428.

Ando, M.; Toyohara, H.; Shimizu, Y. y Sakaguchi, M. 1991. Validity of a puncture test for evaluating the change in muscle firmness of fish during ice storage. Nippon Suisan Gakk, 57: 2341.

Banga, A.; Alonso, A.; Gallardo, J. y Pérez-Martin, R. 1993. Mathematical modelling and simulation of the thermal processing of anisotropic and non-homogeneous conduction-heated canned foods: Application to canned tuna. J Food Eng, 18: 369-387.

Bertullo, V. 1975. Tecnología de los productos y subproductos de pescados, moluscos y crustáceos. (1ra. ed). Buenos Aires: AR. Hemisferio Sur.

Cárdenas, C.; Córdoba, J.; Cohaila, L. y Gonzales, E. 1979. Características físicas y química de las principales especies marinas para consumo humano. (Informe $\mathrm{N}^{\mathrm{o}}$ 52). Callao, Perú: IMARPE.

Choi, Y. y Okos, M. 1986. Effects of temperature and composition on the thermal properties of foods. Food Engineering and Process Applications, (1): 93-101. 
London, UK: Elsevier.

Duran, A.; Erdemli, U.; Karakaya, M. and Yilmaz, M. 2008. Effects of slaughter methods on physical, biochemical and microbiological quality of rainbow trout Oncorhynchus mykiss and mirror carp Cyprinus carpio filleted in pre-, in- or post-rigor periods. Fish Sci, 74: 1146-1156.

Hatae, K.; Tamari, S.; Miyanaga, K. and Matsumoto, J. 1985. Species difference and changes in the physical properties of fish muscle as freshness decreases. B Jpn Soc Sci Fish, 51: 1155-1161.

Huidobro, A y Tejada, M. 1993. Revisión: Propiedades de hidratación del músculo de pescado. Rev Esp Cienc Tecnol Aliment, 33(4): 365-381.

Huss, H. 1988. Fresh fish-quality and quality changes. FAO, Fisheries Series, $N^{\circ}$ 29. Roma, Italia: FAO/ DANIDA, Training Programme on Fish Technology and Quality Control.

Ibarz, A.; Barbosa, G.; Garza, S. y Gimeno, V. 2000. Métodos experimentales en la ingeniería alimentaria. (1ra. ed.). Zaragoza, España: Acribia.

Iwamoto, M.; Yamanaka, H.; Watabe, S. and Hashimoto, K. 1987. Effect of storage temperature on rigor-mortis and ATP degradation in plaice Paralichthys olivaceus muscle. J Food Sci, 52(6): 1514-1517.

Kreith, F. y Bohn, M. 2001. Principios de transferencia de calor. (6ta. ed.) DF, México: Thomson International.

Kumbhar, B.; Agarwal, R. y Das, K. 1981. Thermal properties of fresh and frozen fish. Int J Refrig, 4(3): 143146.

Lind, I. 1991. The measurement and prediction of thermal properties of food during freezing and thawing-A review with particular reference to meat and dough. $J$ Food Eng, 13: 285-319.

Llerena, T. y Nué P. 2002. Efecto de la temperatura de almacenamiento en refrigeración sobre el desarrollo del rigor mortis de la tilapia gris (Oreocromis niloticus). Anales Cientificos (Universidad Nacional Agraria La Molina), 53: 479-488.
Meffert, H. 1984. COST 90: Results of an international project on thermal properties. Rev Int Froid, 7(1): 21-26.

Ochoa, O.; Amézquita, A. and Chejne, F. 2006. Thermophysical properties of meat-A review. Dyna 73(148): 103-118.

Radhakrishnan, S. 1997. Measurement of thermal properties of seafood. (Mg Sc Thesis). Blacksburg, USA: Virginia Polytechnic Institute and State University.

Suárez, H.; De Francisco, A.; Beirão, L.; Pardo, S. y Cortés, M. 2007. Pérdida de textura post mortem de la carne de pescado durante el almacenamiento en frío. Acta Biol Colomb, 12(1): 3-18.

Suzuki, T. 1987. Tecnología de las proteínas de pescado y krill. (1ra. ed.) Zaragoza, España: Acribia.

Tachibana, K.; Misima, T. and Tsuchimoto, M. 1993. Changes of ultrastructure and cytochemical $\mathrm{Mg}^{2+}$-ATPase activity in ordinary muscle of cultured and wild red sea bream during storage in ice. B Jpn Soc Sci Fish, 59: 721727.

Tejada, M.; Huidobro, A. y Fouad Mohamed, G. 2003. Comparison of gilthead sea bream (Sparus aurata) and hake (Merluccius merluccius) muscle proteins during iced and frozen storage. J Sci Food Agric, 83(2), 113-122.

Toyohara, H. y Shimizu, Y. 1988. Relation of the rigor mortis of Fish Body and the texture of the muscle. B Jpn Soc Sci Fish, 54: 1795-1798.

Wallapapan, K.; Sweat, V.; Diehl, K. y Engler, C. 1986. Thermal properties of porous foods. (M.R. Okos, ed.). Physical and chemical properties of foods, pp. 78119. ASAE, MI. 